\title{
Traumatic arterial dissection in open-heart surgery
}

\author{
A. F. MATAR A ${ }^{1}$ AND D. N. ROSS . \\ From the National Heart Hospital and Institute of Cardiology, London
}

During the past few years there have been an increasing number of reports in the medical literature of cases of traumatic dissection as a result of cannulation of the femoral or iliac arteries and retrograde perfusion during openheart surgery (Jones, Vetto, Winterscheid, Dillard, and Merendino, 1960; Elliott and Roe, 1965). Over the past two years (October 1963 to October 1965) in 160 open-heart operations performed at the National Heart Hospital, five cases of traumatic arterial dissections were encountered with an incidence of $3 \cdot 1 \%$.

\section{CASE REPORTS}

CASE 1 F. W. (Hospital no. 1032) A man aged 60 years presented with a five years' history of angina on effort, dyspnoea on exertion, and paroxysmal nocturnal dyspnoea. Previously he had been asymptomatic, though a heart murmur was heard at the age of 25 years.

On examination there was well-marked arcus senilis. B.P. $110 / 65 \mathrm{~mm}$. Hg. There were physical signs of rigid aortic stenosis with minimal regurgitation.

A chest radiograph showed marked post-stenotic dilatation of the ascending aorta and calcification in the region of the aortic valve.

An aortogram showed severely stenosed and immobile aortic cusps and minimal-to-moderate aortic regurgitation. It was not possible to enter the left ventricle.

Operation Homograft replacement of the aortic valve was carried out under normothermic cardiopulmonary bypass with arterial return into the left external iliac artery. The excised valve was rigid and calcified with a triangular orifice measuring $1 \times 1 \times 1$ cm.

On the ninth post-operative day the patient developed ventricular tachycardia and generalized convulsions, but was resuscitated. An early diastolic murmur was detected at that time.

Forty-five days after operation the patient suddenly collapsed while sitting in a chair in the ward and died in spite of immediate attempts to resuscitate him.

'Present address: Harefield Hospital, Harefield, Middlesex
Necropsy A $5 \mathrm{~mm}$. gap was present due to deh1scence of sutures between the homograft and the aortic wall. There was mild atheromatous change in ? the ascending aorta, the arch becoming severe in $\dot{\infty}$ the descending aorta, especially below the renal $N$ arteries, with some ulceration and calcification. 을 There was an extensive dissection with a double lumen extending from the left subclavian to the left $c$ common iliac artery. The false lumen was endothelialized and contained no clot (Fig. 1).

CASE $2^{2}$ A. E. (Hospital no. 7126) A man aged $59 \vec{\varphi}$ years presented with a five years' history of angina on effort which was getting progressively worse. He also complained of dyspnoea on exertion and attacks of paroxysmal nocturnal dyspnoea for three years.

On examination the B.P. was $130 / 80 \mathrm{~mm}$. Hg. There were physical signs of rigid aortic stenosis and minimal regurgitation.

A chest radiograph showed the heart was of normal size. There was moderate calcification in the region of the aortic valve. No dilatation or calcification of the aorta was seen.

Operation On beginning bypass after cannulation of the left external iliac artery the arterial line pressure rose alarmingly, indicating obstruction. The cannula was withdrawn and the right iliac artery was cannulated.

When the ascending aorta was clamped its proximal portion did not decompress. Incision of the ascending aortic wall revealed a double-barrelled chamber with dissection almost to the origins of the coronary arteries. The aortic valve was excised and replaced $D$ with a homograft, and the dissection space was closed proximal to the aortic clamp by a continuous circum- N
ferential suture inserted through all coats.

The heart subsequently took over its work-load $N$ without difficulty. However, 12 hours later the diastolic pressure fell suddenly from $70 \mathrm{~mm}$. $\mathrm{Hg}$ to $\sigma$ zero without any change in the systolic pressure. No diastolic murmur could be heard. A retrograde $\stackrel{0}{\subset}$ aortogram via the right femoral artery was attempted, $\mathbb{D}$ but the catheter could not be manipulated past the aortic knuckle. A cine-aortogram after injection of $T$ dye at this level demonstrated considerable narrowing of the true aortic lumen. There was a soft tissue $\overparen{D}$ shadow of the dissection lying beside the lumen.

${ }^{2}$ This case has been reported in detail (Dongaonkar, 1966) 

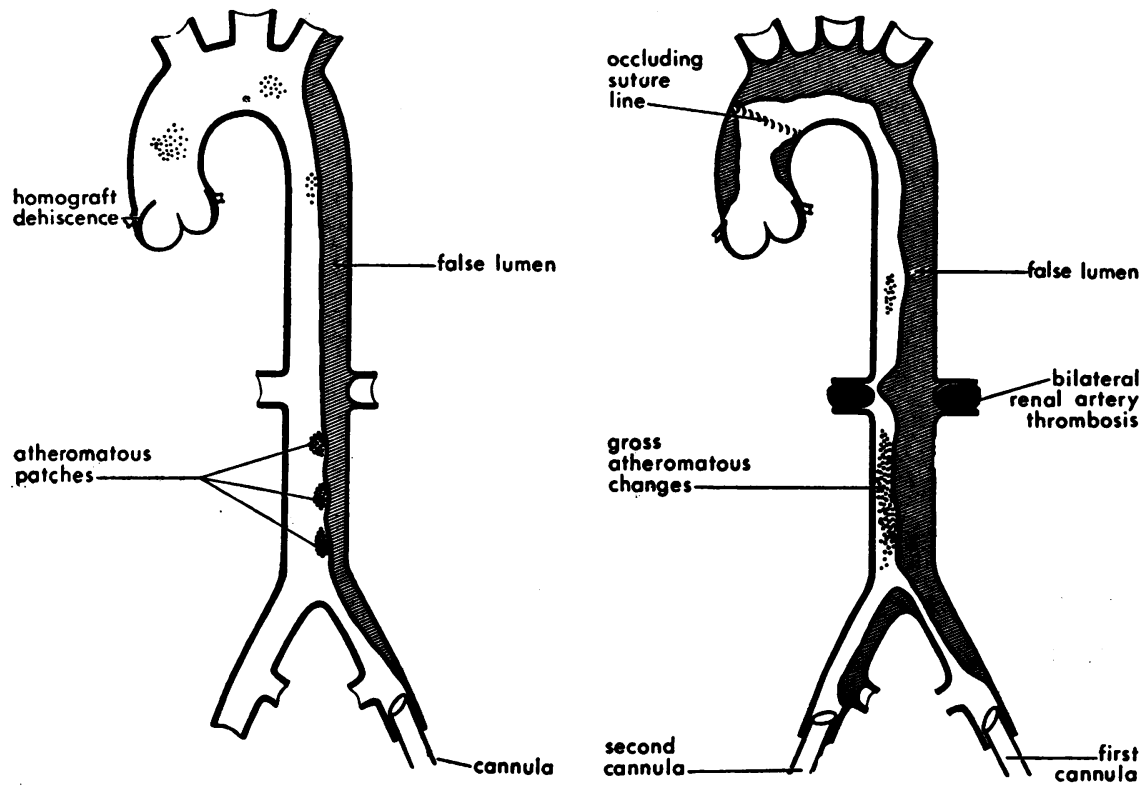

FIG. 1

FIG. 2

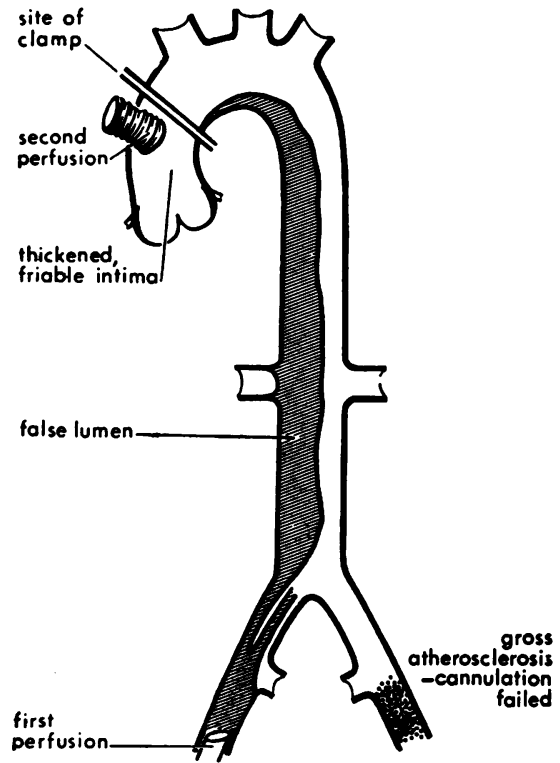

FIG. 3

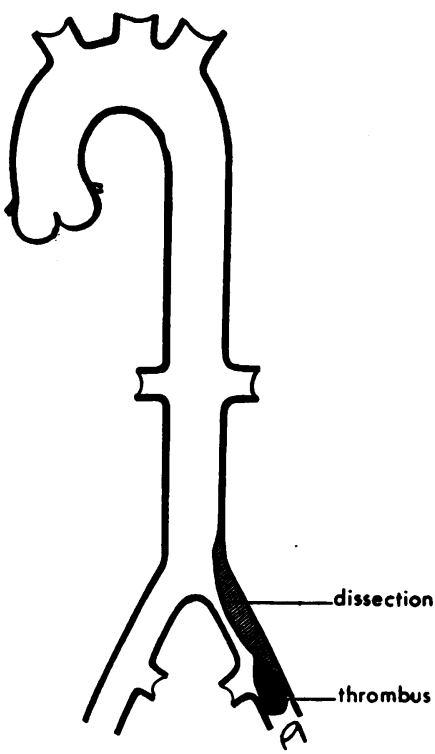

FIG. 4

FIGS 1 to 4 show the post-mortem findings in four fatal cases.

FIG. 1. Case 1. There is some doubt that the dissection was recent, as the false lumen was epithelialized. FIGS 2 AND 3. Cases 2 and 3. Death was evidently due to the dissection.

FIG. 4. Case 4. The dissection was only localized and was not responsible for the patient's death. 
Flow in this area was very sluggish, but there was no regurgitation of the dye into the left ventricle. An injection at the level of the renal arteries showed adequate filling of both vessels from the true lumen, with the left renal artery crossing the dissection. There appeared to be no flow down the left common iliac artery.

No further operative intervention seemed to be justified in view of the extent of the dissection and the fact that there was no evidence of dehiscence past the aortic valve.

On the third post-operative day the patient suddenly collapsed with ventricular fibrillation and resuscitation failed.

Necropsy Examination revealed no evidence of dehiscence of the homograft. The aorta showed widespread atheroma with calcification, ulceration, and thrombosis. The traumatic dissection had spread to include both iliac arteries, the whole length of the descending aorta, and the arch up to the occluding suture line. Both renal arteries were occluded by compression of the dissecting haematoma and thrombosed. Both kidneys showed evidence of massive recent infarction (Fig. 2).

CASE 3 F. R. (Hospital no. 8702) A woman aged 54 years presented with a history of dyspnoea on effort for two years. She had also had two syncopal attacks, which were related to exertion.

On examination the B.P. was $140 / 105 \mathrm{~mm}$. $\mathrm{Hg}$. Physical signs of dominant aortic stenosis with moderate aortic regurgitation were found.

A chest radiograph showed the heart size to be within normal limits. There was some prominence of the ascending aorta. The aortic valve was heavily calcified and there was also some calcification in the left coronary artery.

A left ventricular cine-aortogram showed thickened, rigid, and irregular aortic valve cusps and a severely stenosed valve orifice with moderate aortic regurgitation. The coronary arteries appeared to fill satisfactorily.

Operation When the left common iliac artery was exposed it was found to be grossly atherosclerotic and cannulation was impossible. The right common iliac artery was therefore exposed and, although it was similarly affected, it was possible to cannulate this vessel. As the possibility of dissection was anticipated the line pressure was watched carefully while bypass was started. When the aorta was opened its intima was found to be thickened and friable. The aortic valve was bicuspid, heavily calcified, and disorganized. It was excised and replaced with a freezedried homograft and the aortotomy was closed. On removal of the aortic clamp it was noticed that the pressure in the ascending aorta remained low at 30 to $40 \mathrm{~mm}$. $\mathrm{Hg}$, whereas the pressure in the descending aorta as measured by needle puncture was 150 $\mathrm{mm}$. Hg. The heart action started to deteriorate due to poor coronary perfusion. The body was therefore $\overrightarrow{\bar{F}}$ cooled to $30^{\circ} \mathrm{C}$. and the circulation was then arrested. A finger in the ascending aorta confirmed the presence of dissection up to the point of application of the aortic clamp proximal to the innominate artery. Perfusion was reinstituted through a tubular piece of teflon sewn into the aortotomy incision. This resulted in immediate restoration of heart action, and it was possible to re-warm the patient and wean her off bypass. Good aortic pressures were maintained for 10 minutes, after which there was a rapid and progressive rise in the central venous pressure with a gradual decline in the systemic pressure. Resuscitation failed.

Necropsy examination revealed displacement of an intact tube of intima and media of the right external iliac artery for a distance of $5 \mathrm{~cm}$. from the site of insertion of the perfusion cannula up to the orifice of the common iliac artery. The displaced tube was bent into the common iliac artery and dissection extended from that point up to the site of the clamp on the ascending aorta (Fig. 3).

CASE 4 T. M. (Hospital no. 8542) A man aged 48 years presented with a history of breathlessness on exertion for 15 years and intermittent attacks of congestive cardiac failure for the past 10 years following recurrent chest infections.

When seen he was extremely disabled by breathlessness, orthopnoea, and attacks of paroxysmal nocturnal dyspnoea.

On examination the B.P. was $120 / 75 \mathrm{~mm}$. Hg. There were physical signs of dominant mitral regurgitation associated with mitral stenosis, aortic stenosis, and aortic regurgitation. The liver was palpable, firm, and non-pulsatile, with its lower border two fingerbreadths below the costal margin. The spleen was not enlarged. There was no oedema of the ankles. There was a marked expiratory wheeze and a prolonged expiratory phase.

A chest radiograph showed a large heart, left atrial and left ventricular enlargement, calcification of the mitral valve, and mild pulmonary venous congestion.

An aortogram showed minimal aortic incompetence. The movement of the aortic cusps was restricted, but there was no appreciable dilatation of the ascending aorta. The coronary arteries showed no evidence of disease.

Operation Homograft replacement of the mitral valve was performed under hypothermic $\left(30^{\circ} \mathrm{C}\right.$. $)$ cardiopulmonary bypass with arterial return to the left external iliac artery. The heart initially took over its load efficiently, but there was torrential loss of blood from all the myocardial incisions, which could not be controlled. Effective heart action could not be maintained and the patient died on the table.

At necropsy the homograft was still firmly in position with no evidence of dehiscence. The abdominal portion of the aorta showed minimal atheroma. A traumatic cannulation thrombus was present in the 
left external iliac artery for a distance of $5 \mathrm{~cm}$. Immediately above the thrombus the artery had dissected as far as its origin, extending for a distance of $3 \mathrm{~cm}$. into the terminal aorta, where there was a re-entry ostium (Fig. 4).

CASE 5 G. O. (Hospital no. 8341) A woman aged 52 years gave a history of a heart murmur which had been heard in childhood, and consequently she had attended a school for physically handicapped children.

She had been asymptomatic until the age of 48 , when she started to develop substernal pain radiating to the right side of the neck, shoulder, and arm.

She had also been troubled with fatigue and breathlessness on exertion.

On examination the B.P. was $130 / 90 \mathrm{~mm}$. Hg. There were physical signs of severe aortic stenosis with minimal regurgitation.

A chest radiograph showed a normal-sized heart. There was marked post-stenotic dilatation, a heavily calcified aortic valve, and clear lung fields.

An aortogram revealed a heavily calcified, rigid, and irregular aortic valve with marked post-stenotic dilatation of the aorta. The right coronary artery did not fill, but absence of filling could have been due to streaming.

Operation Replacement of the aortic valve with a Starr-Edwards prosthesis was performed under cardiopulmonary bypass.

The left common iliac artery was cannulated without difficulty, but it was immediately noticed that there was a very poor back-flow into the cannula. Localized dissection of the atherosclerotic artery was diagnosed and the cannula was immediately withdrawn. The diagnosis was confirmed by inspecting the inside of the artery. In view of our previous experience in case 3 no attempt was made to cannulate the right external iliac artery. Instead, we proceeded to direct cannulation of the ascending aorta.

The operation was uneventful and the left common iliac artery was sutured at the end of bypass.
The post-operative recovery was without incident and the patient was discharged home well.

The Table shows that two of the reported patients died as a direct result of traumatic arterial dissection (cases 2 and 3 ). In patient 1 , however, we cannot be certain that the dissection was the direct cause of death. As the false lumen was partly epithelialized and despite the fact that the patient survived for 45 days, it is difficult to be certain whether the dissection was present before the operation or not. The patient also had aortic regurgitation as a result of partial dehiscence of the homograft.

Patient 5 survived because the dissection was discovered at a very early stage and direct aortic perfusion was instituted instead of making further attempts at cannulation of the opposite external iliac artery, in which case the outcome would probably have been similar to that in cases 2 and 3.

TECHNIQUE OF CANNULATION OF THE ASCENDING AORTA

As a result of our experience with the above cases we adopted the technique of cannulation of the ascending aorta (Nuñez and Bailey, 1959; DeWall and Levy, 1963).

The site chosen for cannulation, near the root of the innominate artery, is prepared by clearing the loose part of the adventitia and the pericardial reflection. This step is important in order to avoid the cannula being caught in this loose areolar tissue during its insertion. It also facilitates suture of the aortic incision at the end of the procedure.

A no. 00 purse-string silk suture is inserted through the adventitia and media, avoiding, if

T A B L E

SUMMARY OF FIVE CASES OF TRAUMATIC ARTERIAL DISSECTION

\begin{tabular}{|c|c|c|c|c|c|c|c|}
\hline Case & Age & Sex & $\begin{array}{l}\text { B.P. } \\
(\mathrm{mm} . \mathbf{H g})\end{array}$ & Diagnosis & Extent of Dissection & When Diagnosed & Outcome \\
\hline F.W. & 60 & $\mathbf{M}$ & $110: 65$ & $\begin{array}{l}\text { Acquired calcific } \\
\text { A.S. + minimal } \\
\text { A.R. }\end{array}$ & $\begin{array}{l}\text { Left subclavian to left common } \\
\text { iliac artery }\end{array}$ & At necropsy & $\begin{array}{l}\text { Died } 45 \text { days after } \\
\text { operation in V.F. }\end{array}$ \\
\hline A.E. & 59 & $\mathbf{M}$ & $130: 80$ & $\begin{array}{l}\text { Acquired calcific } \\
\text { A.S. + minimal } \\
\text { A.R. }\end{array}$ & $\begin{array}{l}\text { From both iliac arteries to origin } \\
\text { of coronary arteries including } \\
\text { all branches of the arch and } \\
\text { both renal arteries }\end{array}$ & $\begin{array}{l}\text { L.C.I. early; aorta, when } \\
\text { opened at the beginning } \\
\text { of operation }\end{array}$ & $\begin{array}{c}\text { Died } 3 \text { days after } \\
\text { operation in V.F. }\end{array}$ \\
\hline $\begin{array}{r}3 \\
\text { F.R. }\end{array}$ & 54 & $\mathbf{F}$ & $140 ' 105$ & $\begin{array}{l}\text { Congenital calcific } \\
\text { A.S. + moderate } \\
\text { A.R. }\end{array}$ & $\begin{array}{l}\text { From right external iliac artery } \\
\text { to site of aortic clamp proxi- } \\
\text { mal to the innominate artery }\end{array}$ & $\begin{array}{l}\text { After completion of valve } \\
\text { replacement and closure } \\
\text { of aorta }\end{array}$ & $\begin{array}{l}\text { Died on table in } \\
\text { myocardial failure }\end{array}$ \\
\hline T.M. & 48 & $\mathbf{M}$ & 12075 & $\begin{array}{l}\text { Acquired A.S. }+ \\
\text { A.R. and M.R. }\end{array}$ & $\begin{array}{l}\text { Localized dissection in left } \\
\text { common iliac artery }\end{array}$ & At necropsy & $\begin{array}{l}\text { Died on table from } \\
\text { intractable bleeding }\end{array}$ \\
\hline G.O. & 52 & $\mathbf{F}$ & $130 / 90$ & Congenital A.S. & $\begin{array}{l}\text { Localized dissection in external } \\
\text { iliac artery }\end{array}$ & At time of cannulation & Survived \\
\hline
\end{tabular}

A.S. = aortic stenosis;

A.R. = aortic regurgitation

L.C.I. = left common iliac artery;

M.R. = mitral regurgitation; 
possible, penetration of the intima. The ends of the suture are threaded through a snare. For extra safety a second purse-string can be added. The cannula we use is a 7/32 in. Simplastic gamma-ray sterilized vena caval cannula with one terminal and four side holes. This size is suitable for all adults. In children we use a smaller size, depending on the diameter of the aorta, usually $5 / 32$ in. The cannula should allow retrograde flow between it and the aortic wall in order to perfuse the vessels of the head adequately. A stainless steel connexion with a side-tap is attached to the other end of the cannula. The end of the connexion is plugged while the tap is left open, to allow escape of air at the time of cannulation, and is switched off by the assistant in time to avoid blood loss.

A lateral occlusion clamp is applied to the aortic wall in order to isolate the selected cannulation area without obstructing the aortic flow. A vertical incision in line with the aorta is made in the area demarcated by the purse-string using a no. 12 curved blade. As the clamp is released the cannula is inserted for a distance of 7 to 10 $\mathrm{cm}$. so as to lie in the arch of the aorta. As soon as the blood fills the cannula the tap is closed by the assistant and the purse-string is tightened. The cannula is secured in position by tying it to the snare with nylon tape and is further secured to the arm of the retractor with another tape. The cannula is then connected to the arterial line after air has been excluded by gradually releasing a clamp applied to the cannula while it is being connected.

At the end of perfusion the side occlusion clamp is reapplied after the cannula has been removed. The aortotomy is then sutured in two layers and the purse-string suture is tightened in order to take the strain off the suture line.

\section{DISCUSSION}

Traumatic arterial dissection at the time of cannulation for cardiopulmonary bypass has now become a recognized hazard of this procedure (Williams and Johnson, 1964).

Elderly or hypertensive patients with cystic medial necrosis of the aortic walls are particularly prone to this hazard (Manley, 1964). All our patients were above 45 years of age, but none of them was hypertensive.

Although many authorities deny that atherosclerosis is a primary cause of spontaneous arterial dissection and insist that the coexistence of atherosclerosis and dissection is not necessarily related (Hudson, 1965), it seems to be an $\overline{\overline{\bar{S}}}$ important factor in dissection of traumatic origin.?

Dissection usually occurs due to the presence흘 of atheromatous areas in the cannulated artery $\bar{c}$ or more proximally in the path of a retrograde $\stackrel{\mathbb{Q}}{\mathbb{Q}}$ stream. A high velocity retrograde jet may disrupto diseased intima at the tip of the cannula at the $\vec{P}$ time of cannulation or later during the perfusion. This is particularly likely if the cannula changes $\vec{\omega}$ its position and the jet becomes directed towardso the arterial wall. The diseased intima can also become disrupted at sites remote from the tip of the cannula, e.g., at the aortic bifurcation or the abdominal aorta. Elliott and Roe (1965) reportedos two cases of arterial dissection during cardio- -0 pulmonary bypass in which the dissection did not? take place at the time of cannulation but occurred after the patients had been on bypass for? a considerable period of time. At necropsy it waș verified that the intimal tear took place in ar diseased part of the aorta at its bifurcation and well away from the tip of the cannula, which was inserted into the common femoral artery. The predisposition to dissection was due to atherosclerosis in one case and to cystic medial necrosis in the other.

Braunstein (1963) studied the clinical historyo and necropsy records of 35 cases of aortic dis section and found that in 30 cases the outstandinge clinical features were old age, hypertension, and atherosclerosis. The remaining five patients, how ever, were young subjects. The prominent features in the latter group were fragmentation of the elastica and the massive accumulation of mucoid substance in the wall of the aorta.

Hudson (1965) maintains that there are two distinct types of patient with dissection: youngerd patients with fragmentation of the elastic tissue in the media; and older patients with cystic necrosis of the muscle of the media.

From the above it becomes evident that the danger of dissection not only exists in the older age-group but is a hazard in the younger patiento as well.

A group particularly prone to dissection ares patients with dilated arteries. This was shown in a necropsy study of the total aortic area in 336 patients (Mitchell, Schwartz, and Zinger, 1964). It is also well known that patients with Marfan's syndrome are particularly prone to dissection.

Other conditions which have been reported too increase the susceptibility to dissection are calcific aortic stenosis, whether congenital or acquiredळ (Petch, 1952; Hudson, 1965; Heath, Edwards 20 and Smith, 1958), and bacterial endocarditis. 
Traumatic arterial dissection is just one of the hazards to which cannulation of the femoral or iliac arteries subjects patients. Cases of retroperitoneal haematoma have been described (Van der Woude and Iticovicci, 1962). DeWall and Levy (1963) maintain that this type of cannulation leads to almost total ischaemia of $20 \%$ of the total body weight throughout the duration of bypass, with subsequent flooding of the circulation with accumulated metabolites at the time of decannulation, in addition to pooling of blood in the dilated limb vessels.

There are also delayed complications. We have encountered one case of claudication following cannulation of limb arteries. The patient required a second operation to disobliterate the thrombosed external iliac artery four months after the bypass procedure.

There are also likely to be a number of cases of localized dissections which pass unnoticed. Some may remain silent, but many of the puzzling clinical pictures which follow this type of surgery may be due to ischaemia of various organs as a result of healing of a dissection. Healing dissections have been reported to cause paraplegia, steatorrhoea, large bowel obstruction, renal complications, cerebral ischaemia, aortic regurgitation, and myocardial infarction. A healed dissection may also be the source of thrombo-embolism thought to be originating from the prosthetic valve. Long survival also allows the possibility of bacterial endocarditis of the dissection (Robinson, 1958).

Since we adopted the technique of direct cannulation of the ascending aorta we have used it in $128^{3}$ cases without complication. As a result we are now convinced of the superiority of this technique. It has the advantage of not extending the operative field. It is easy and permits the rapid introduction of almost any size of cannula and an equally simple and time-saving decannulation. The risks related to atherosclerosis of the limb vessels and the difficulties of their cannulation are avoided. A forward flow from a large-size cannula is also less likely to disrupt the diseased intima at sites remote from the tip of the cannula.

Kerth (1965) reported a case in which dissection occurred during bypass and the patient was saved by removing the cannula from the external iliac artery and replacing it in the aorta. Following bypass a decompression window was created. The

'Up to 20 July 1966. The technique has been used in 70 more cases up to 7 December 1966, still without complication patient survived and an angiogram showed a persistent double aortic lumen.

A further advantage of this technique is that the perfusion site is continuously under vision throughout the duration of bypass and any leak can be detected immediately. There is also some evidence that perfusion of the brain and heart is better and, in hypothermic perfusion, the cooled blood is primarily directed to the important visceral organs and then to the muscle mass (DeWall and Levy, 1963).

\section{SUMMARY}

Five cases of traumatic arterial dissection due to cannulation of the iliac artery during cardiopulmonary bypass are reported. The technique of aortic cannulation is described. The immediate and delayed complications of cannulation of the limb vessels as well as the advantages of direct cannulation of the aorta are discussed.

We wish to thank Mrs. Helen Forsyth for the typing and Mrs. Dawbarn for the drawings.

\section{REFERENCES}

Beresford, O. D. (1951). The clinical diagnosis of dissecting aneurysm of the aorta. Brit. med. J., 2, 397.

Braunstein, H. (1963). Pathogenesis of dissecting aneurysm. Circulation, 28, 1071 .

DeWall R. A. and Levy, M. J. (1963). Direct cannulation of the ascending aorta for open-heart surgery. J. thorac. cardiovasc. Surg., 45, 496.

Dongaonkar, P. P. (1966). Acute aortic dissection following cannulation of the iliac artery. Brit. Heart J., 28, 431.

Elliott, D. P., and Roe, B. B. (1965). Aortic dissection during cardiopulmonary bypass. J. thorac. cardiovasc. Surg., 50, 357.

Heath, D., Edwards, J. E., and Smith, L. A. (1958). The rheologic significance of medial necrosis and dissecting aneurysm of the ascending aorta in association with calcific aortic stenosis. Mayo Clin. Proc., 33, 228.

Hudson, R. E. B. (1965). Cardiovascular Pathology, p. 512. Arnold, London.

Jones, T. W., Vetto, R. R., Winterscheid, L. C., Dillard, D. H., and Merendino, K. A. (1960). Arterial complications incident to cannulation in open-heart surgery, with special reference to the cannulation in open-heart surgery, with

Kerth, W. (1965). J. thorac. cardiovasc. Surg., 80, 362.

Manley, G. (1964). Histology of the aortic media in dissecting aneurysms. J. clin. Path., 17, 220.

Mitchell, J. R. A., Schwartz, C. J., and Zinger, A. (1964). Relation ship between aortic plaques and age, sex, and blood pressure. Brit. med. J., 1, 205.

Nuñez, L. E., and Bailey, C. P. (1959). New method for systemic arterial perfusion in extracorporeal circulation. J. thorac. Surg. 37, 707.

Petch, C. P. (1952). Congenital aortic aneurysm with valvular stenosis and dissecting aneurysm. Brit. Heart J., 14, 147.

Robinson, G. L. (1958). Double-barrelled aorta with bacterial endarteritic vegetations. Ibid., $20,571$.

Van der Woude, R., and Iticovicci, H. (1962). Retroperitoneal haemorrhage as a complication of femoral artery cannulation for extracorporeal circulation. A case report. J. thorac. cardiova sc. Surg. corporeal 540 .

Williams, K. R., and Johnson, J. (1964). Aortic dissecti on after femoral artery cannulation. Arch. Surg., 89, 663. 